\title{
NuSTAR ground calibration: The Rainwater Memorial Calibration Facility (RaMCaF)
}

\author{
Brejnholt, Nicolai; Christensen, Finn Erland; Jakobsen, Anders Clemen; Hailey, Charles J.; Koglin, \\ Jason E.; Blaedel, Kenneth L.; Stern, Marcela; Thornhill, Doug; Sleator, Clio; Zhang, Shuo \\ Total number of authors: \\ 15
}

Published in:

Proceedings of SPIE - The International Society for Optical Engineering

Link to article, DOI:

10.1117/12.894659

Publication date:

2011

Document Version

Publisher's PDF, also known as Version of record

Link back to DTU Orbit

Citation (APA):

Brejnholt, N., Christensen, F. E., Jakobsen, A. C., Hailey, C. J., Koglin, J. E., Blaedel, K. L., Stern, M., Thornhill, D., Sleator, C., Zhang, S., Craig, W. W., Madsen, K. K., Decker, T., Pivovaroff, M. J., \& Vogel, J. K. (2011). NuSTAR ground calibration: The Rainwater Memorial Calibration Facility (RaMCaF). Proceedings of SPIE - The International Society for Optical Engineering, 8147(1), 81470l-9. https://doi.org/10.1117/12.894659

\section{General rights}

Copyright and moral rights for the publications made accessible in the public portal are retained by the authors and/or other copyright owners and it is a condition of accessing publications that users recognise and abide by the legal requirements associated with these rights.

- Users may download and print one copy of any publication from the public portal for the purpose of private study or research.

- You may not further distribute the material or use it for any profit-making activity or commercial gain

- You may freely distribute the URL identifying the publication in the public portal 


\title{
NuSTAR ground calibration: The Rainwater Memorial Calibration Facility (RaMCaF)
}

\author{
Nicolai F. Brejnholt $^{a}$, Finn E. Christensen ${ }^{a}$, Anders C. Jakobsen ${ }^{a}$, Charles J. Hailey ${ }^{b}$, Jason E. \\ Koglin $^{b}$, Kenneth L. Blaedel ${ }^{b}$, Marcela Stern ${ }^{b}$, Doug Thornhill ${ }^{b}$, Clio Sleator ${ }^{b}$, Shuo Zhang ${ }^{b}$ \\ ,William W. Craig ${ }^{c}$, Kristin K. Madsen $^{d}$,Todd Decker ${ }^{e}$, Michael J. Pivovaroff $^{e}$, Julia K. Vogel ${ }^{e}$ \\ ${ }^{a}$ DTU Space, Technical University of Denmark, Copenhagen, Denmark \\ ${ }^{b}$ Columbia University Astrophysics Laboratory, New York City, USA \\ ${ }^{c}$ Space Science Laboratory, University of California, Berkeley, USA \\ ${ }^{d}$ California Institute of Technology, Pasadena, USA \\ ${ }^{e}$ Lawrence Livermore National Laboratory, Livermore, USA
}

\begin{abstract}
The Nuclear Spectroscopic Telescope Array (NuSTAR) is a NASA Small Explorer mission that will carry the first focusing hard X-ray $(5-80 \mathrm{keV})$ telescope to orbit. The ground calibration of the three flight optics was carried out at the Rainwater Memorial Calibration Facility (RaMCaF) built for this purpose. In this article we present the facility and its use for the ground calibration of the three optics.
\end{abstract}

Keywords: X-ray optics calibration, NuSTAR, hard X-rays, multilayers

\section{INTRODUCTION}

The Nuclear Spectroscopic Telescope Array (NuSTAR) ${ }^{1}$ is a NASA Small Explorer mission scheduled for launch in early 2012. The ground calibration of the two flight optics (and a third flight spare) was carried out at the Rainwater Memorial Calibration Facility for X-ray optics $(\mathrm{RaMCaF})^{2}$ at Nevis Laboratories in New York.

NuSTAR will be the first focusing hard X-ray telescope on-orbit and has undergone an extensive ground calibration to address the unique challenges associated with the low graze angles, multilayer coatings and broad bandpass. Absolute calibration of the NuSTAR instrument will be performed on-orbit while the ground calibration campaign focused on a relative calibration across the entire pass band.

Each optic is comprised of 2376 pieces of multilayer coated glass substrates, distributed over 133 concentric shells. Individual substrates span $30^{\circ}$ (layer $69-133$ ) or $60^{\circ}$ (layer $1-68$ ) and have radii ranging from $54.4 \mathrm{~mm}$ to $191 \mathrm{~mm}$. Figure 1 summarizes the details of the build. The coatings, utilizing Pt/C (layer $1-89$, referred to as the inner groups) and $\mathrm{W} / \mathrm{Si}$ (layer $90-133$, referred to as the outer groups), are optimized for energies between $5-80 \mathrm{keV}$. The $\mathrm{Pt} / \mathrm{C}$ is coated according to seven multilayer recipes, each optimized for the relevant radii/grazing angle, while three different recipes were used for the $\mathrm{W} / \mathrm{Si}$ layers. ${ }^{3}$

In order to carry out detailed analysis of the ground calibration data a thorough understanding of the optic response, calibration approach and calibration facility is required. Section 2 briefly introduces the calibration facility. An extensive introduction to the calibration approach focusing on the hardware utilized will follow in Sec. 3. An in-depth description of the acquired data and early results can be found elsewhere in these proceedings. ${ }^{4}$ Section 4 will discuss ongoing efforts to improve the optic response model through flight substrate and multilayer coating qualification.

Further author information: N.F.B.: E-mail: nicolai@space.dtu.dk, Telephone: +45 35325791

Optics for EUV, X-Ray, and Gamma-Ray Astronomy V, edited by Stephen L. O'Dell, Giovanni Pareschi, Proc. of SPIE Vol. 8147, 81470I - @ 2011 SPIE · CCC code: 0277-786X/11/\$18 · doi: 10.1117/12.894659 




Figure 1. Detailed top view of a full sextant of an optic. Reproduced from the optics design document

\section{CALIBRATION FACILITY}

RaMCaF was constructed in an existing underground tunnel, neighboring the NuSTAR optics' assembly room. The facility was built specifically for the NuSTAR flight optics' ground calibration and for prototype and multilayer coating witness sample testing. A hard X-ray continuum up to $100 \mathrm{keV}$ is produced by a high power X-ray tube located $160 \mathrm{~m}$ upstream from the optic hutch. The source-optic distance and the nearly $11 \mathrm{~m}$ optic-focal plane distance is under medium vacuum $(0.1$ Torr $)$. When the beam exits the vacuum pipe into the optic hutch its diameter is nearly $0.5 \mathrm{~m}$. Additional details regarding the facility can be found in a dedicated publication. ${ }^{2}$

The workhorse detector of the facility is an Ortec High-Purity Germanium (Ge) detector. The Ge detector is well-understood based on an absolute calibration against the RHESSI ${ }^{5}$ flight spare detector and a detailed mass model. It has excellent high-energy quantum efficiency, but the nearly $5 \mathrm{~cm}$ thick crystal is not ideal for low energy measurements with a Bremsstrahlung spectrum due to energy redistribution. For this purpose a prototype SDD was used. Work is in progress to build a detailed mass model of the SDD through cross-calibration with the Ge detector and radioactive sources. The SDD is under development at the Institut de Recherche en Astrophysique et Planétologie (IRAP) and is based on a large area chip by PNDetector GmbH. ${ }^{6}$

In addition to the above work, ongoing efforts focused on modeling the X-ray source and beam are in progress to allow for end-to-end validation of the calibration results. The X-ray source model is based on the detailed Ge detector model and direct beam measurements, while a campaign to study the beam uniformity was carried out prior to calibration. The campaign revealed that the beam has a $5 \%$ vertical gradient over its full extent and that the vacuum windows introduce non-uniformity on the order of $5 \%$ below $8 \mathrm{keV}$.

\section{CALIBRATION CAMPAIGN}

The baseline calibration approach for the relative effective area of the NuSTAR optics was similar to past soft X-ray telescope ground calibrations. ${ }^{7}$ It consisted of full aperture exposures (full flood) and sub-aperture (sub-group) exposures. Table 1 contains an overview of measurement types and their purpose. The sub-group exposures enable quasi-parallel illumination by co-aligning the optic axis and the divergent X-ray beam at the 


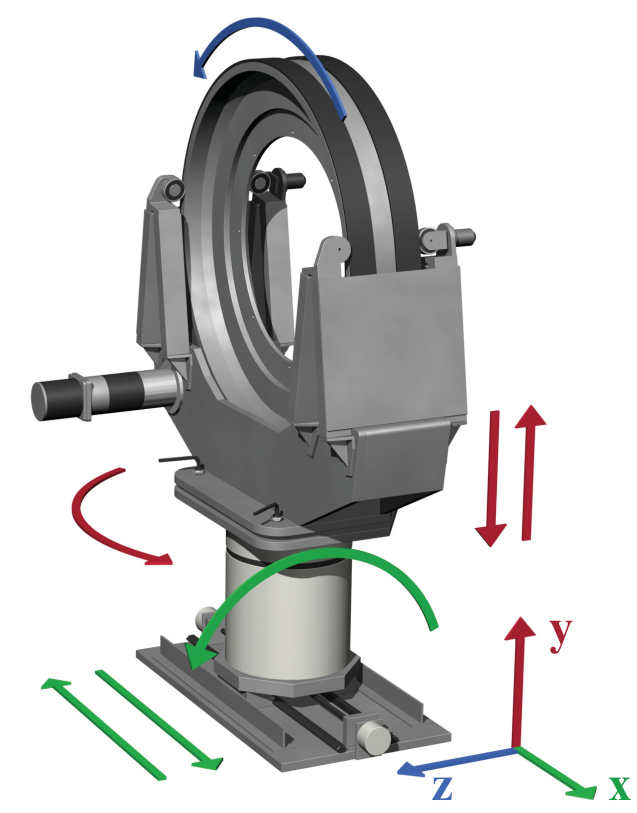

Figure 2. Bench with five degrees of freedom used for the calibration of focusing X-ray optics, most recently the NuSTAR flight optics. Rotation around the X (pitch, green), Y (yaw, red) and Z (roll, blue) axes is available as well as translation in $\mathrm{X}$ and $\mathrm{Y}$. $\mathrm{Z}$ values are positive in the direction of the focal plane at RaMCaF.

relevant radii, thus simulating an infinite source distance. In this configuration both on- and off-axis throughput and multilayer response were measured for all sub-groups, imitating on-orbit conditions. The wedge type measurements were only carried out on a single $60^{\circ}$ segment of the optic, while the binocular type was done for several sub-groups. Binocular measurements were carried out by illuminating two $60^{\circ}$ azimuthal segments in turn, off-set $180^{\circ}$ in azimuth from each other. The horizontal difference in the focal plane location of the maximum throughput for the two segments describes how far out of focus the optic is.

Manipulation of the optic during calibration was achieved using a bench custom-built for testing focusing X-ray optics. ${ }^{8}$ Figure 2 indicates how the bench can pitch, yaw and roll the optic around its respective axes as well as translate it perpendicular to the X-ray beam (vertically and horizontally).

The majority of the work was carried out using the non-imaging detectors mentioned in Sec. 2 and a slit unit, but an imaging detector was also used for X-ray alignment and PSF investigations. The imaging detector is a novel electron multiplying CCD (EMCCD) developed by RMD Inc. ${ }^{9}$ The EMCCD is coupled to a thin micro columnar scintillator film $(\mathrm{CsI}(\mathrm{Tl}))$ via a fiber optic taper and has a pixel pitch of $16 \mu \mathrm{m}$ and an imaging area of $8.2 \times 8.2 \mathrm{~mm}^{2}$. For the purpose of the NuSTAR calibration $2 \times 2$ on-chip binning and a 3:1 taper was utilized to increase image area. A future publication will treat the use of the EMCCD during calibration in greater detail. ${ }^{2,10}$

\subsection{Optic alignment}

Coarse alignment of the optic was carried out using an optical alignment telescope pointed along the X-ray axis. Alignment of the critical pitch and yaw of the optic to the facility axes was performed by adjusting the struts indicated in Figure 3a. The alignment telescope was operated in auto-collimation mode off a flat mirror installed against a diamond-turned reference surface of the optic mandrel. The coarse optical alignment was estimated to be better than $1^{\prime}$. An electronic autocollimator was installed at the opposite end of the optic reflecting against a second, similarly installed flat mirror. The autocollimator acted as a fixed reference throughout calibration, providing arcsecond-level knowledge of the relative movement of the optic in pitch and yaw. 
Table 1. Overview of measurement types and purposes. The radial and azimuthal extent of the individual measurements are given as well as the hardware utilized. Refer to Sec. 3.3 Table 2 for details on the hardware abbreviations. This table also contains bow-tie (BT) and lead curtain (LC) used to vary the azimuthal extent of the coarse aperture stop aperture and blocking one or the other side during binocular measurements, respectively.

\begin{tabular}{|l|l|l|l|l|}
\hline Type & Radial layers & Azimuth extent & Hardware & Purpose \\
\hline Full flood & All & $360^{\circ}$ & FFAS & Alignment and throughput \\
\hline Sub-group & $6-8$ & $360^{\circ}$ & FFAS+AP & $\begin{array}{l}\text { Detailed study of single subgroups' } \\
\text { throughput and relative multilayer re- } \\
\text { sponse }\end{array}$ \\
\hline Sub-group & $6-8$ & $60^{\circ}$ & CAS+AP+FAS & $\begin{array}{l}\text { Quasi-parallel throughput and relative } \\
\text { multilayer response (on- and off-axis) }\end{array}$ \\
\hline Wedge & Inner groups & $2^{\circ}$ & CAS+BT+OFAS & $\begin{array}{l}\text { Detailed study to determine average } \\
\text { PSF, throughput and relative multi- } \\
\text { layer response versus azimuth angle }\end{array}$ \\
\hline Wedge & Outer groups & $2^{\circ}$ & $\begin{array}{l}\text { Cetailed study to determine average } \\
\text { PSF, throughput and relative multi- } \\
\text { layer response versus azimuth angle }\end{array}$ \\
\hline Binocular & $6-8$ & $2 \times 60^{\circ}$ & FFAS+LC+AP & \begin{tabular}{l} 
Best focus search and image features \\
\hline
\end{tabular}
\end{tabular}

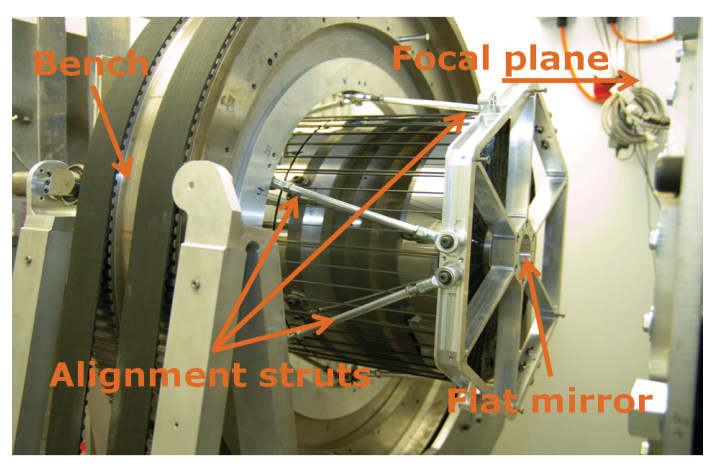

(a)

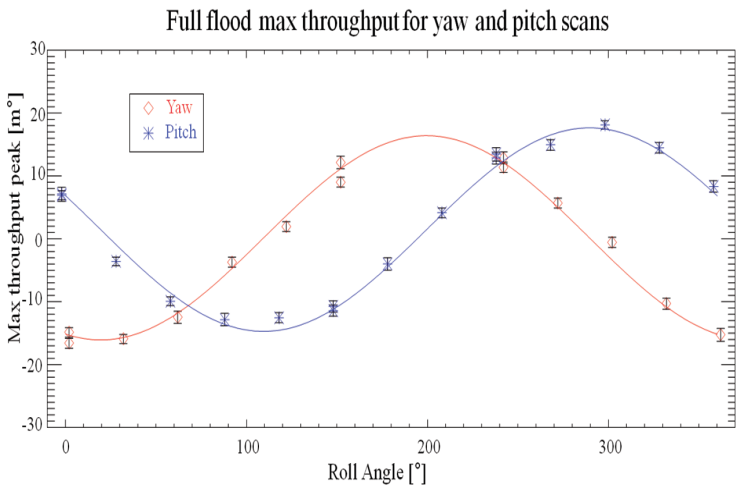

(b)

Figure 3. (a) Photo of a flight optic installed in the bench. Three of the alignment struts on the optic spider fixture are indicated along with the location of the flat mirror used for optical alignment. The electronic autocollimator uses a similar mirror placed at the opposite end of the optic. Focal plane direction is indicated (b) Summary of results from rolling FM1 in increments of $30^{\circ}$ and scanning pitch and yaw in turn over a broad range to determine maximum throughput. The precession is fit (line) by a wedge in the autocollimator mirror of order $58^{\prime \prime} \pm 0.7^{\prime \prime}$.

X-ray alignment consisted of finding maximum throughput at several roll positions by scanning pitch and yaw over a wide range. Figure $3 \mathrm{~b}$ shows a summary of the results for flight model 1 (FM1). The precession corresponds to a wedge in the autocollimator mirror of order $58^{\prime \prime} \pm 0.7^{\prime \prime}$. The optic was adjusted based on these results throughout the calibration campaign for on- and off-axis measurements.

\subsection{Optic bend}

After X-ray alignment sub-group data demonstrated a slight, but significant, asymmetry in the PSF as shown in Fig. 4a. Gravitational bending was initially suspected to be the cause, but lack of rotational symmetry indicated that additional components were present. Through ray tracing it was found that overconstraining the optic with the alignment struts (ref. to Fig. 3a) had induced a bend along the optical axis. Figure 4b shows a ray trace with the optic bent according to the proposed model. The effect was confirmed by loosening the alignment struts 


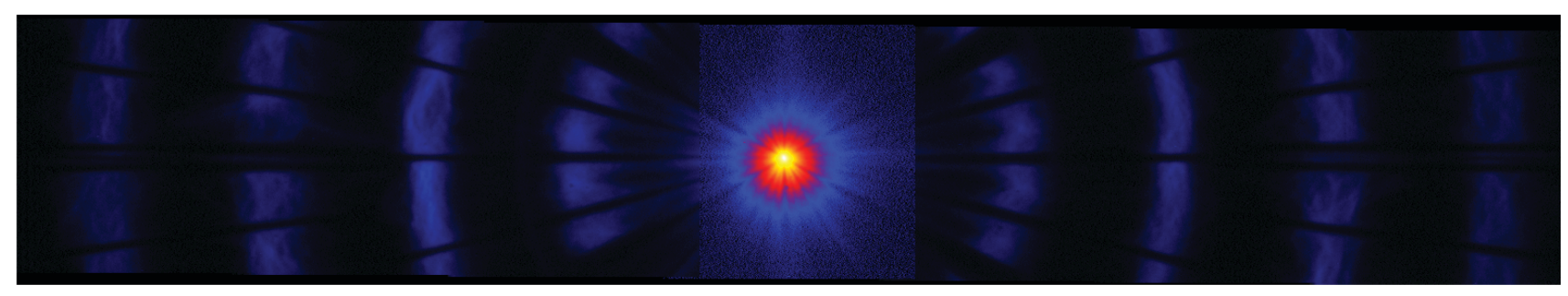

(a)

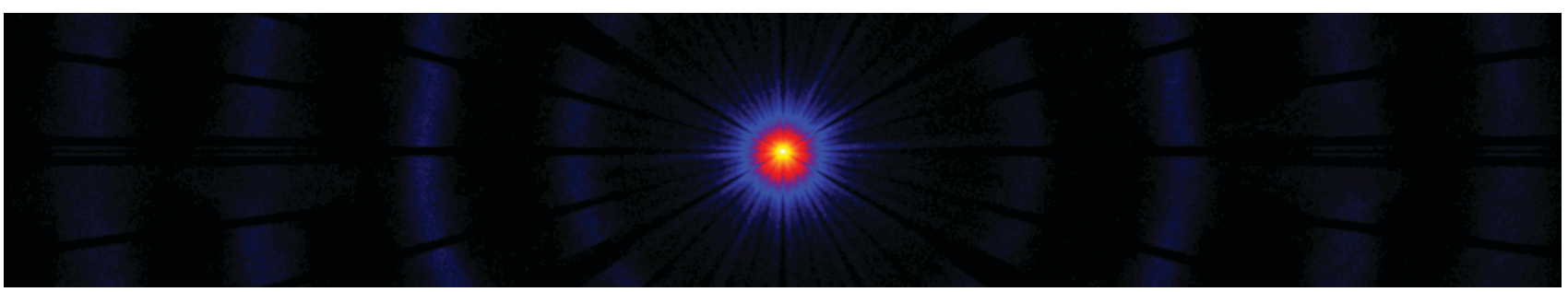

(b)

Figure 4. (a) Composite image acquired by translating the EMCCD horizontally. A slight asymmetry of the spot is apparent. Work is in progress to correct for detector effects which can be observed in the high intensity central image (b) Ray trace simulation of an optic suffering from a $0.003^{\circ}$ bend in the vertical plane.

Table 2. Overview of hardware types and purpose. Table 1 and Figure 7 indicates location and use of hardware items. All items were manufactured from $4 \mathrm{~mm}$ lead backed by aluminum.

\begin{tabular}{|l|l|l|}
\hline Type & Abbreviation & Description \\
\hline Coarse aperture stop & CAS & $0^{\circ}$ to $70^{\circ}$ coarse aperture for use with all sub-groups \\
\hline Full flood aperture stop & FFAS & $360^{\circ}$ aperture for full flood illumination \\
\hline Fine aperture stop & FAS & $60^{\circ}$ precision aperture for use with all sub-groups \\
\hline Inner fine aperture stop & IFAS & $60^{\circ}$ precision aperture for use with inner sub-groups \\
\hline Outer fine aperture stop & OFAS & $30^{\circ}$ precision aperture for use with outer sub-groups \\
\hline Aperture plate & AP & Eight plates, containing aperture patterns for twenty sub-groups \\
\hline Aperture ring & AR & Six plates that mounts to AP selecting specific sub-group(s) \\
\hline
\end{tabular}

and observing a reduced asymmetry. During final integration and alignment at Jet Propulsion Lab (JPL) the range of motion at either end of both optics was controlled such that no significant stress was put into either optic module. ${ }^{11}$ No measurable asymmetry is expected on-orbit.

\subsection{Calibration hardware}

Table 2 contains an overview of the hardware used for the full flood and sub-group measurements. Along with Table 1 it describes the setups used for both optics' calibration. The initial alignment and throughput measurements were done using only the full flood aperture stop (Figure 5a). In this configuration aperture plates (Figure 5b) were also used, and aperture rings when required, to map out contributions from individual sub-groups. The individual sub-groups were also measured with the coarse aperture stop (i.e. limited azimuthal extent). This configuration further utilized the fine aperture stops (Figure 6). Figure 7 shows where the hardware is installed in the optic hutch relative to the optic. Note that the aperture plate is physically mounted to the optic mandrel and spider fixture and the aperture rings in turn mount onto the aperture plates. The fine aperture stop is mounted on the bench such that it follows the optic in all motions except roll. 


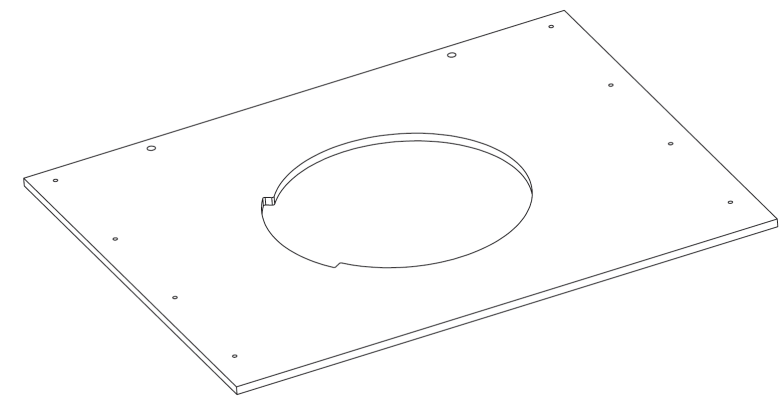

(a)

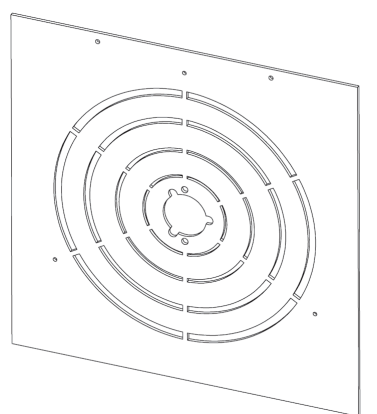

(b)

Figure 5. (a) Full flood aperture stop. The coarse aperture stop is a replica of this, but with a $70^{\circ}$ aperture instead of $360^{\circ}$ (b) Eight aperture plates exists with anywhere from one to four sub-groups on a single plate. The one shown here contain sub-group 1, 9, 15 and 19, exposing half of recipe 1, 5, 8 and 10 layers when installed. A further sub-selection among these sub-groups can be carried using aperture rings which are mounted directly onto the AP.

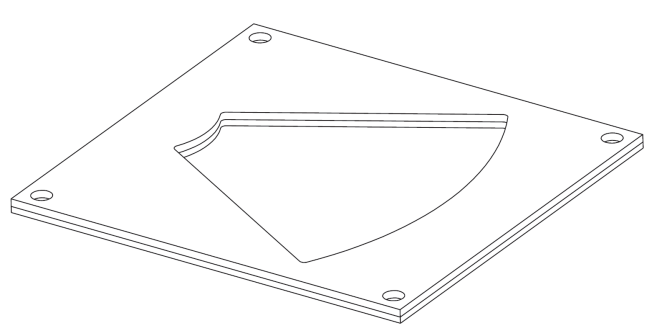

(a)

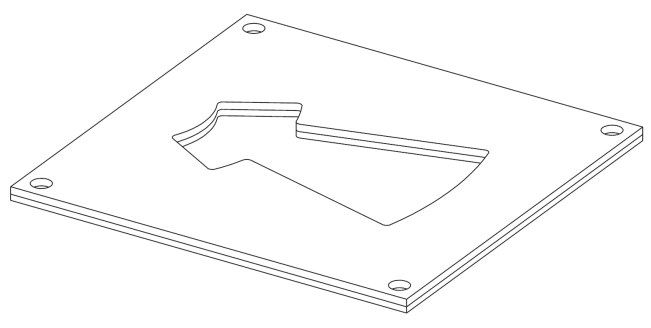

(b)

Figure 6. (a) Fine aperture stop with $60^{\circ}$ opening for all sub-groups (b) Fine aperture stop with $60^{\circ}$ opening for inner groups and $30^{\circ}$ opening for outer groups

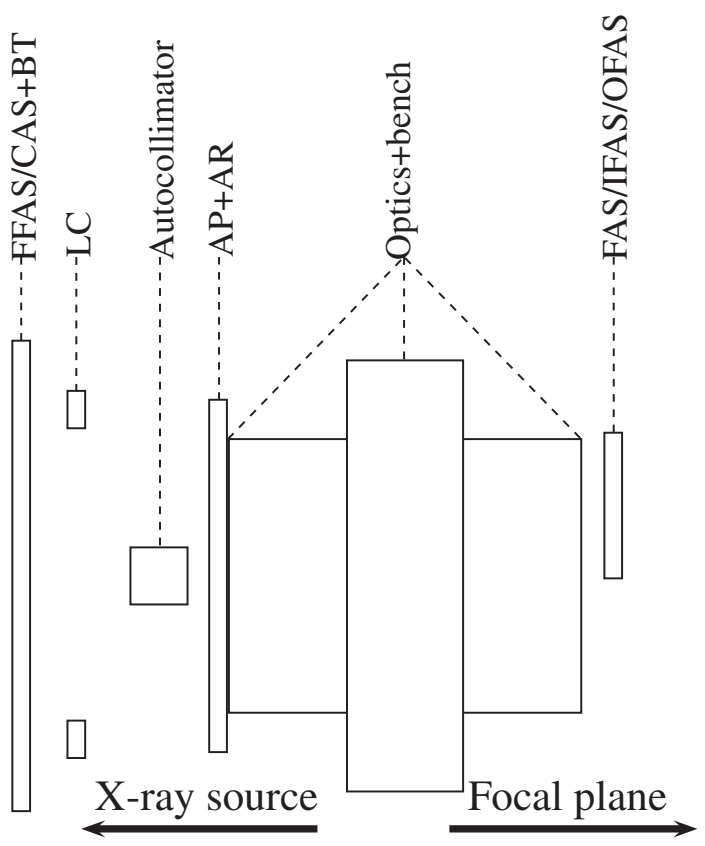

Figure 7. Sketch of the optic hutch layout. Backslash symbols indicate that hardware items are mutually exclusive while plus signs indicate simultaneous installation is possible. 


\section{OPTIC RESPONSE MODEL IMPROVEMENTS}

Detailed modeling of the optic response requires knowledge of the as-coated multilayer coating, mounted substrate figure and other scattering mechanisms. Here we focus on the investigations carried out on flight coated witness samples (Sec. 4.1), but will give brief mention of the ongoing efforts in the other areas.

The mounted substrate figure was measured during production using a linear variable differential transformer (LVDT) providing figure estimates on length scales between $5 \mathrm{~mm}$ and $200 \mathrm{~mm}$. Experience from HEFT has shown that these data can be used to estimate the core of the PSF for an X-ray optic, ${ }^{12,13}$ which was exploited in early analysis of FM1 and FM2. However, in order to estimate the full PSF, more detailed investigations of the scattering mechanisms below the $5 \mathrm{~mm}$ length scale must be carried out. Ongoing efforts include analysis of X-ray synchrotron scatter data acquired from flight coatings in 2010 at NSLS (length scales up to a couple of hundred micrometers, limited by mounting figure error) as well as surface metrology of flight-spares using AFM ${ }^{14}$ (length scales from tens of nanometers to a couple of micrometers) and optical surface profiling (length scales from a micrometer to a millimeter). The height data obtained through these studies were used to compute power spectral densities (PSD). The PSDs are well-fit by power-law models, and the fractal nature of the surface height errors suggests that X-ray scattering from these mirrors can be described by the formalism developed by Church and Takacs. ${ }^{15,16}$ Including the actual multilayer parameters, determined from the witness sample campaign (ref. to Sec. 4.1) and using ray trace code, presented in these proceedings, ${ }^{17}$ the refined optics response model will be validated through comparison with the calibration data.

\subsection{Witness sample campaign}

The NuSTAR flight recipes were optimized using a Figure of Merit developed for the HEFT balloon mission. ${ }^{18,19}$ Frequent coating calibration runs were carried out to ensure uniform coating rates in the nearly 200 flight coatings. Nevertheless run to run variations of the coating rate are expected as well as non-uniformity over the curved slumped glass within a single coating run. Efforts to map out the non-uniformity of the coating deposition as a function of chamber location and an in-depth look at the overall results of the witness sample campaign are presented elsewhere in these proceedings. ${ }^{20}$

To monitor the run to run variations, flat Silicon wafers were included in each coating run and grazing incidence reflectivity measurements subsequently carried out with a pencil beam setup at RaMCaF. The setup is illustrated in Fig. 8. The first slit unit limits the beam to $0.1 \times 4 \mathrm{~mm}^{2}$ while the detector slit unit is wide open $\left(15 \times 8 \mathrm{~mm}^{2}\right)$. Measurements are carried out with a nominal fixed grazing angles of $0.22^{\circ}(\mathrm{Pt} / \mathrm{C})$ or $0.24^{\circ}$ $(\mathrm{W} / \mathrm{Si})$. Figure 9a shows data and fit from a representative $\mathrm{Pt} / \mathrm{C}$ coated witness sample. Reflectivity is derived from measuring the reflected beam as shown in Fig. 8, followed by translating the bench and multilayer out of the beam and the second slit unit and detector back into the direct beam.

The thickness of the individual bilayers of the NuSTAR multilayer coating design is described by a power law which was used in initial attempts to fit the data with an IMD $^{21}$ based fitting routine. Although results were fair, the large data set available indicated that systematic deviations were present. High-resolution transmission electron microscopy (HRTEM) carried out on witness samples showed a small increase in the heavy-to-light material ratio $(\Gamma)$ in the upper bilayers and indicated that the first few top bilayers deviated significantly from the design values. Implementing this in the fitting routine significantly improved the goodness of fits. In Fig. $9 \mathrm{~b}$ the deviation from nominal recipe thickness is plotted for the initial fifteen layers of a NuSTAR recipe. It should be noted that only the initial six layers have been fit individually. Beyond this point we lose our ability to resolve single layer contributions to the relative reflectivity of the multilayer. Additional HRTEM measurements are being carried out to further investigate the deviations. 


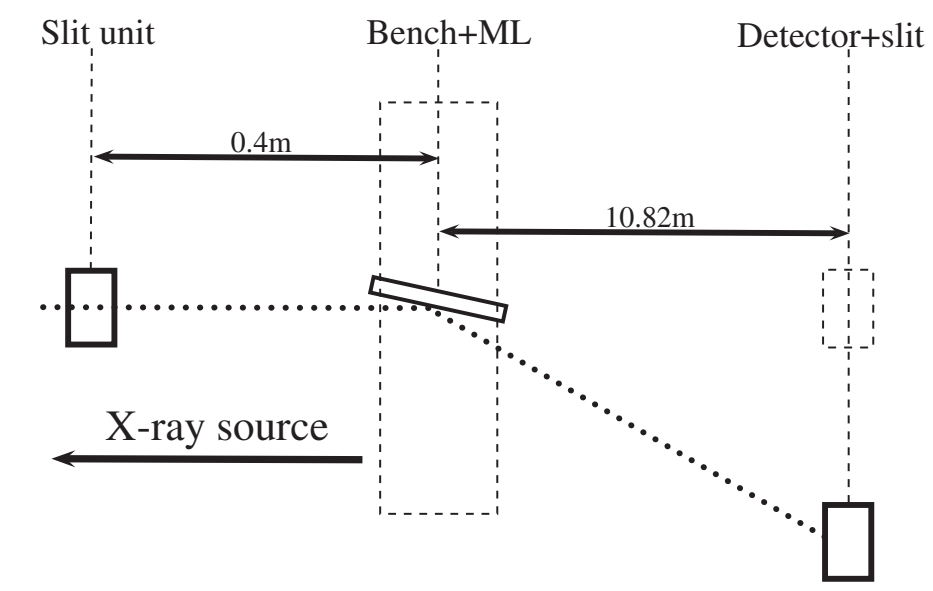

Figure 8. Sketch of the witness sample measurement setup. The electronic autocollimator (not indicated in sketch) is situated between the first slit unit and the bench. The location of the detector+slit unit during direct beam acquisition is outlined.

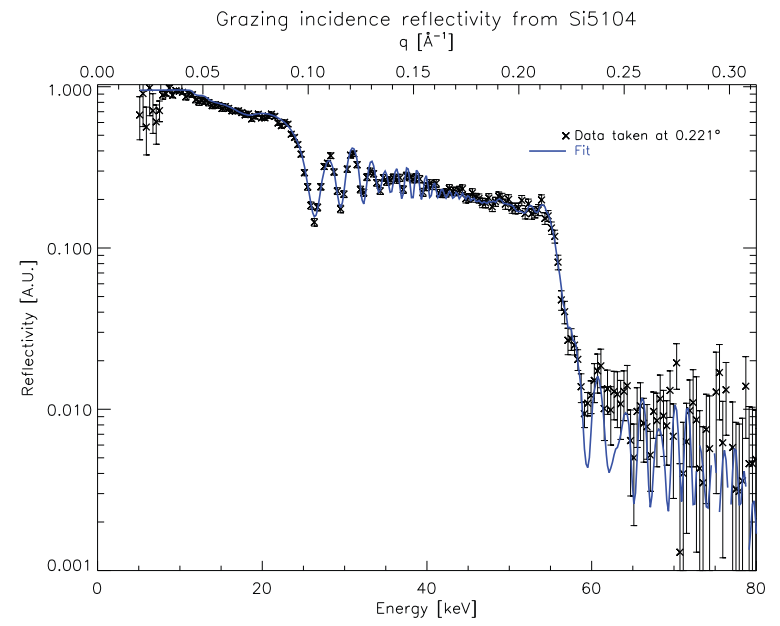

(a)

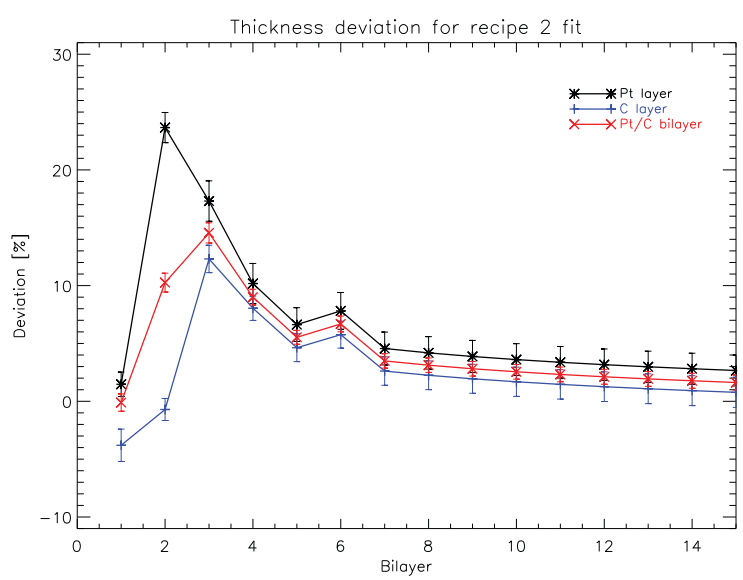

(b)

Figure 9. (a) Witness sample from NuSTAR flight recipe $2(\mathrm{Pt} / \mathrm{C})$ coating run. Data acquired at RaMCaF and fit carried out using reflectivity function from IMD implemented in custom fit routine (b) Shows thickness deviation from designed bilayer thickness as a function of bilayer for the top fifteen bilayers. The data set is comprised of the average deviation of the fifteen recipe 2 flight coating runs. 


\section{ACKNOWLEDGMENTS}

The NuSTAR mission is funded by NASA through contract number NNG08FD60C. Part of this work was funded by the Technical University of Denmark (DTU Space). Surface metrology (AFM and optical surface profiling ) was performed under the auspices of the U.S. Department of Energy by Lawrence Livermore National Laboratory (LLNL) under Contract DE-AC52-07NA27344. LLNL contributions also included support from LDRD project 10-SI-007. The prototype SDD was provided by Didier Barret of the Institut de Recherche en Astrophysique et Planétologie (IRAP). The prototype SDD development is funded by IRAP, the French National Center for Scientific Research (CNRS) and the Centre National d'Etudes Spatiales (CNES). The use of the EMCCD X-ray detector for optics calibration was made possible thanks to NASA SBIR Grant NNX11CH32P.

\section{REFERENCES}

[1] Harrison, F. A. et al., "The Nuclear Spectroscopic Telescope Array (NuSTAR)," in [Space Telescopes and Instrumentation 2010: Ultraviolet to Gamma Ray Optics], Proc. SPIE 7732, 77320S (2010).

[2] Brejnholt, N. F. et al., "The Rainwater Memorial Calibration Facility (RaMCaF) for X-ray optics," X-ray Optics and Instrumentation 2011 (2011).

[3] Hailey, C. J. et al., "The Nuclear Spectroscopic Telescope Array (NuSTAR): Optics Overview and Current Status," in [Space Telescopes and Instrumentation 2010: Ultraviolet to Gamma Ray Optics], Proc. SPIE 7732, $77320 \mathrm{~T}(2010)$.

[4] Koglin, J. et al., "NuSTAR ground calibration," These proceedings (2011).

[5] Lin, R. P. et al., "The Reuven Ramaty High-Energy Solar Spectroscopic Imager (RHESSI) mission," Proc. SPIE 5171, 38-52 (2004).

[6] Schlosser, D. M. et al., "Expanding the detection efficiency of silicon drift detectors," Nuclear Instruments and Methods in Physics Research Section A: Accelerators, Spectrometers, Detectors and Associated Equipment 624, 270-276 (2010).

[7] Gondoin, P. et al., "Calibration of the first XMM Flight Mirror Module II - Effective Area," in [X-Ray Optics, Instruments, and Missions], Proc. SPIE 3444, 278-289 (1997).

[8] Christensen, F. E. et al., "X-ray Calibration of the SODART flight telescopes," in [Grazing Incidence and Multilayer X-ray Optical Systems], Proc. SPIE 3113, 294-306 (1997).

[9] Thacker, S. C. et al., "Development of a high-speed CT imaging system using an EMCCD Camera," in [Physics of Medical Imaging], Proc. SPIE 7258, 725846 (2009).

[10] Vogel, J. K. et al., "Application of an EMCCD Camera for Calibration of Hard X-ray Telescopes," IEEE Proceedings 2011 Article in preparation.

[11] Craig, W. W. et al., "Fabrication and metrology of the NuSTAR flight optics," These proceedings (2011).

[12] Koglin, J. et al., "Development and Production of Hard X-ray Multilayer Optics for HEFT," Proc. SPIE 4851, 607-618 (2003).

[13] Koglin, J. et al., "Production and Calibration of the first HEFT Hard X-ray Optics module," Proc. SPIE 5168, 100-111 (2004).

[14] Soufli, R. et al., "Atomic force microscopy characterization of Zerodur mirror substrates for the extreme ultraviolet telescopes aboard NASA's Solar Dynamics Observatory," Applied Optics 46, 3156-3163 (2007).

[15] Church, E. L. et al., "Specification of surface figure and finish in terms of system performance," Applied Optics 32, 3344-3353 (1993).

[16] Church, E. L. et al., "Specification of glancing- and normal-incidence X-ray mirrors," Optical Engineering 34, 353-360 (1995).

[17] Westergaard, N. J. et al., "MT-RAYOR: a versatile raytracing tool for X-ray telescopes," These proceedings (2011).

[18] Harrison, F. A. et al., "Development of the High-Energy Focusing Telescope (HEFT) balloon experiment," in [X-Ray Optics, Instruments, and Missions], Proc. SPIE 4012, 693-699 (2000).

[19] Mao, P. H. et al., "Evaluation and optimization of multilayer designs for astronomical x-ray telescopes using a field-of-view- and energy-dependent figure of merit," Proc. SPIE 4138, 126-133 (2000).

[20] Christensen, F. E. et al., "NuSTAR flight coatings - what did we really do," These proceedings (2011).

[21] Windt, D. L. et al., "IMD - Software for modeling the optical properties of multilayer films," Computers in Physics , 360-370 (1998). 